\title{
Genetical analysis of intrapopulational variation in olfactory response in Drosophila melanogaster
}

\author{
E. Alcorta* and \\ J. Rubio
}

Departamento de Genética, Universidad de Oviedo, 33071 Oviedo, Spain.

Genetical study of olfactory response to ethyl alcohol ( 8 per cent) has been carried out on inbred lines extracted from a natural population using biometrical and chromosome analyses. A full diallel analysis was made in seven endogamic lines (3 high, 3 low and 1 intermediate in response values). Our results show a highly significant $(P<0.001)$ additive component of variance and a less significant $(P<0.05)$ directional dominance component suggesting overdominance and some endogamic depression. No significant maternal or reciprocal components of variation appear in our population. Two chromosome substitution assays among 3 inbred lines of extreme olfactory response add up information to the results of diallel analysis. The differences in response are due to chromosome III with an additive effect highly significant $(P<0 \cdot 001)$ in both studies. The other major chromosomes do not show any effect themselves but some interactions with chromosome III are significant. They can explain the directional dominance that we find in the diallel study. Analysed olfactory variation in response to ethanol can be considered as unspecific in relation to various chemical compounds.

\section{INTRODUCTION}

Olfactory response forms a part of the behaviour patterns which are responsible for survival and reproduction of Drosophila. In spite of this, the genetics of olfaction has been little studied.

At present, studies on genes which control this response and relations among them are almost completely limited to behaviour genetics work using induced mutants (Kikuchi, 1973; Rodrigues and Siddiqi, 1978; Aceves-Piña and Quinn, 1979). There has been little work on the parts of genome responsible for differences in olfactory response and the relations among them in natural populations. Only selection (Becker, 1970; Hoffmann, 1983) and partial chromosome substitution (Fuyama, 1978) experiments have been carried out. Response obtained by selection indicated additive variance in natural populations for olfactory behaviour to different chemicals. Moreover, Becker (1970) suggested that alleles for insensitivity to repellents found by him could be dominants. On the other hand, Fuyama (1978) located variability in response to esters and ketones on the right arm of the chromosome II. Differences among isochromosomal lines for the chromosome II were due to polygenic factors with additive effect.

The objective of the present study was to survey, through classic methods of quantitative genetics, the genetical effects associated with phenotypic differences on olfactory response to ethyl alcohol ( 8 per cent) in a natural population. We could then compare the kind of relation among genes and the importance of this character in fitness (Breese and Mather, 1960; Kearsey and Kojima, 1967), as has been made for other behavioural traits. Response to ethyl alcohol has been chosen for genetic analysis, as its variability in a natural population from Sandiche (Spain) shows up more clearly than reponses to other stimuli we have assayed in the same population.

\section{MATERIAL AND METHODS}

\section{Olfactometry}

The olfactometer used was a $Y$-maze with two ways of choice (fig. 1). A tube with a filter paper impregnated with either $0.5 \mathrm{ml}$ odorant or $0.5 \mathrm{ml}$ distilled water was placed at the end of each arm. 

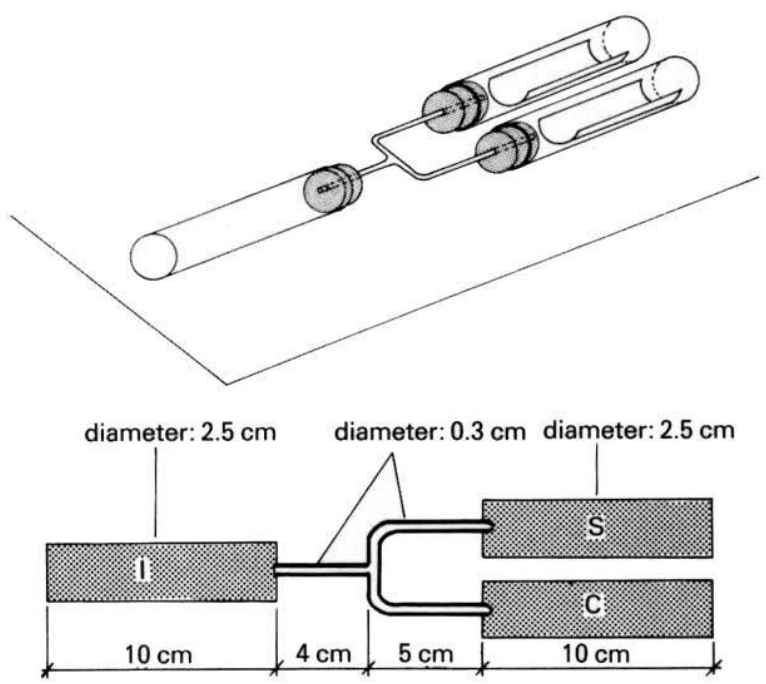

Figure 1 Olfactometer. (1) initial tube, (S) stimulus tube, with a piece of filter paper impregnated with $0.5 \mathrm{ml}$ of odorant and $(\mathrm{C})$ control tube with $0.5 \mathrm{ml}$ of distilled water.

Mazes were set horizontally to the floor to avoid geotropic effects and they, were lighted homogeneously. Flies tested were always $603-4$ days old females which had been starved for 24 hours before the test. The olfactory index was: $\mathrm{IO}=$ No. flies in $\mathrm{S} /($ No. in $\mathrm{S}+$ No. in $\mathrm{C})$, where $\mathrm{S}$ indicates stimulant and $\mathrm{C}$ indicates control. IO values are between 0 and 1 . Olfactory index measured in this way involves percentages over different totals. This fact makes the statistical analysis by normal parametric methods more difficult, since data error depends on the number of individuals considered (Snedecor and Cochran, 1984).

The weighted and non parametric methods of statistical analysis that we used when we studied the IO values on a population have not been used here, because the analysis in this study is more complex. On the other hand, the individuals tested in each run are genetically homogeneous (endogamic lines or F1 between endogamic lines), and the different number of flies considered in various tests is not as important as when the line studied had genetically different individuals.

\section{Flies and stimuli}

In order to make a genetical study of a natural population from Sandiche (Asturias, Spain), which showed variation in olfactory response to ethyl alcohol ( 8 per cent) when we tested 51 isofemale lines, we have obtained inbred lines from it. These inbred lines were obtained from 11 isofemale lines whose olfactory response values represented the complete range of populational distribution. From each isofemale line we set up 15 sublines which were maintained by full brothersister crossing. The 31 lines obtained from all the isofemale lines after 16 generations were considered genetically homogeneous. An olfactory study of them showed response values to ethyl alcohol ( 8 per cent) in the same range of population responses and IO means as 0.3320 and 0.3749 for inbred lines and population respectively. Neither distribution of response values was significantly different when they were compared by a contingency $\chi^{2}$. These facts indicate that the inbreeding process has not assembled gene combinations with phenotypical values more extreme than those of the population and populational distribution could be represented by inbred line distribution.

The response values observed are between 0.5326 and 0.2585 , i.e. in the repellent response area. At this range of responses IO high corresponds to indifferent response and IO low to high repellency. This is to say, a low IO represents more sensitivity to stimulus than a high IO value. From the inbred lines we have chosen several lines for different experiments.

\section{(a) Full diallel analysis}

Seven inbred lines have been utilised. The three with highest response values, 89-1,25-13 and 110-6, the three with lowest IO, 16-7, 45-1 and 45-13 and one intermediate, 25-1. (Lines 25-1 and 25-13 come from the isofemale line with the highest IO in the population and 45-1 and 45-13 from the isofemale line with the lowest IO).

A complete $7 \times 7$ diallel set of crosses among the 7 inbred lines has been carried out. The 49 progeny families obtained by crossing each inbred line to each other and to itself were measured for olfactory response to ethyl alcohol (8 per cent). Four replicate tests of each progeny family were made in 4 blocks of measure (a replicated test of each cross in each block).

The statistical treatment of the data we have used is Hayman's model (1954) for partition of variance among lines in additive, dominant, reciprocal and maternal components. The significance of different components is contrasted with their block interaction (we do not have a measure of error variance with only one replicate for each cross in each block). 


\section{(b) Chromosomal substitution assay}

Three inbred lines, 45-1, 89-1 and 25-13 have been used for two chromosomal substitution experiments, one between 45-1 and 89-1 lines and the others between 45-1 and 25-13. Combinations of the three major chromosomes from both pairs of lines were synthesized following Kearsey and Kojima's (1967) crossing scheme. The inversion chromosomes used were Binscy, $C y O$ and TM3 $S b$ Ser. Each substitution is referred to by three numbers denoting the source ( 4 for 45-1 line, 8 for 89-1 and 2 for 25-13) of the first, second and third chromosome pairs. The letter $\mathrm{X}$ was used to refer to chromosomal heterozygotes.

These two sets of chromosomal combinations were tested with ethyl alcohol ( 8 per cent). We made 10 replicate measures for each combination. The significant effects of chromosomes and chromosomal interactions were studied later for additive and dominant contribution using the genetical model described by Mather and Jinks (1971) and explained for the case of three chromosomes by Albornoz et al. (1987). Parameters which describe additive and dominant effects of the chromosomes as well as interchromosomal interactions are also the same as in Albornoz's work. $D 1$, $d 2$ and $d 3$ represent the additive effects of chromosomes I, II and III; $h 1, h 2$ and $h 3$ represent their corresponding dominance effects and the other 20 parameters describe interchromosomal interactions. Parameters $i$ refer to $d \times d$ or $d \times d \times d$ interactions, $j$ to $d \times h$ (i.e., $j 1.2$, interaction $d 1 \times h 2$ ), $d \times d \times h$ (i.e., $j 12.3$, interaction $d 1 \times d 2 \times h 3$ ) or $d \times h \times h$ (i.e., $j 1.23$ interaction $d 1 \times h 2 \times h 3$ ) interactions and 1 to $h \times h$ or $h \times h \times h$ interactions. The parameter scores are estimated by solving equations obtained by equating observed cell means to their corresponding sum of components. Variances were homogeneous between cells within each substitution test. Errors of the genetical parameters are obtained from the variance-covariance matrix.

\section{(c) Specific or unspecific character of olfactory response}

Lastly, specific or unspecific character of olfactory response differences was analysed using four synthetic lines A, B, C and D. They come from four inbred lines, the two highest (25-13 and 89-1) and the two lowest (16-7 and 45-1) response values.

Synthetic lines were obtained crossing endogamic lines in order to avoid endogamic depression effects. Lines A, B, C and D were the F1 generation of crosses between 16-7 $\times 45-1,45-1 \times 89-1,45-1 \times$ 25-13 and 25-13 $\times 89-1$, respectively. So we expected the maximum difference between lines A (F1 of the two inbred lines with lowest IO) and D (F1 of the two lines with highest IO).

Olfactory response on these lines has been tested to 6 stimuli. For every one we have studied the dose-response curve by testing 5 concentrations (concentrations are expressed on percentages in volume):

ethyl alcohol: $1 \%, 3 \%, 10 \%, 30 \%, 100 \%$, methyl alcohol: $1 \%, 3 \%, 10 \%, 30 \%, 100 \%$, acetaldehyde: $0 \cdot 1 \%, 0 \cdot 3 \%, 1 \%, 3 \%, 10 \%$, benzaldehyde: $0.001 \%, 0.01 \%, 0.03 \%, 0.1 \%$, $0 \cdot 3 \%$,

ethyl acetate: $0 \cdot 1 \%, 0 \cdot 3 \%, 1 \%, 3 \%, 10 \%$, acetone: $0 \cdot 1 \%, 0 \cdot 3 \%, 1 \%, 3 \%, 10 \%$.

Dose-response curves to ethyl alcohol were obtained in order to see whether the observed high IO values, near $\mathrm{IO}=0 \cdot 5$, corresponded to no perception of stimulus or only to sensitivity differences. Moreover, we wanted to characterise the differences in response to this compound among synthetic lines to compare them with the other dose-response curves.

The shape of the dose-response curve was studied by a regression analysis for each line and stimulus. Variance of the data to different concentrations was divided in two components: (a) variance explained by adjustment to a regression line, with 1 degree of freedom and (b) variance of deviation to this regression line, with the other degrees of freedom (Sokal and Rohlf, 1979).

\section{RESULTS}

\section{(a) Diallel analysis}

Olfactory response values to ethyl alcohol ( 8 per cent) on the 49 lines obtained by crossing seven inbred lines in all the possible combinations are shown in table 1. IO means of inbred lines are located on the diagonal of the table. The highest IO values corresponded to inbred lines, 0.4908 and 0.4904 of the $25-13$ and $110-6$ lines respectively, but the lowest IO were not on the diagonal. So, the lowest IO value of inbred lines was 0.1420 of the line $16-7$, but IO became $0.0386,0.0549$ and 0.0803 when this line was crossed with the 45-1 and 45-13 lines. Moreover, IO values of the other crosses were often more extreme than IO values of their parents. These facts indicate the existence of endogamic depression and overdominance.

Partition of variance according to Hayman's model (1954) (table 2) showed that almost all the 
Table 1 Mean values of $\mathrm{IO}$ in the diallel crossing

\begin{tabular}{|c|c|c|c|c|c|c|c|}
\hline lines & $\begin{array}{l}\text { (females) } \\
16-7\end{array}$ & $45-1$ & $45-13$ & $25-1$ & $89-1$ & $110-6$ & $25-13$ \\
\hline \multicolumn{8}{|c|}{ (males) } \\
\hline $16-7$ & $0.1420 \pm 0.0358$ & $0.0549 \pm 0.0184$ & $0.3511 \pm 0.1482$ & $0.1634 \pm 0.0660$ & $0.3227 \pm 0.0517$ & $0.1928 \pm 0.0747$ & $0.3197 \pm 0.0507$ \\
\hline $45-1$ & $0.0386 \pm 0.0056$ & $0 \cdot 2585 \pm 0 \cdot 1363$ & $0 \cdot 1304 \pm 0 \cdot 0883$ & $0.2140 \pm 0.0593$ & $0 \cdot 1476 \pm 0.0525$ & $0.2169 \pm 0.0457$ & $0.2821 \pm 0.0566$ \\
\hline $45-13$ & $0.0803 \pm 0.0313$ & $0.2632 \pm 0.1131$ & $0 \cdot 4861 \pm 0 \cdot 1049$ & $0.2055 \pm 0.0522$ & $0.2310 \pm 0.0772$ & $0.2810 \pm 0.0862$ & $0 \cdot 1843 \pm 0 \cdot 0622$ \\
\hline $25-1$ & $0.1348 \pm 0.0221$ & $0.0518 \pm 0.0122$ & $0.3035 \pm 0.0309$ & $0.2668 \pm 0.0509$ & $0.4137 \pm 0.0172$ & $0.4131 \pm 0.0649$ & $0.4290 \pm 0.0868$ \\
\hline $89-1$ & $0.2150 \pm 0.0711$ & $0.1299 \pm 0.0287$ & $0.1503 \pm 0.0668$ & $0.4821 \pm 0.0563$ & $0.5326 \pm 0 \cdot 1007$ & $0.3672 \pm 0.0515$ & $0.4685 \pm 0.0830$ \\
\hline $110-6$ & $0.1496 \pm 0.0641$ & $0.4183 \pm 0.0753$ & $0 \cdot 3709 \pm 0.1480$ & $0.4420 \pm 0.0673$ & $0.4384 \pm 0.1120$ & $0.4904 \pm 0.0488$ & $0.3910 \pm 0.0768$ \\
\hline $25-13$ & $0.2936 \pm 0.0770$ & $0 \cdot 2175 \pm 0 \cdot 1034$ & $0 \cdot 4021 \pm 0 \cdot 1010$ & $0.4455 \pm 0.0697$ & $0 \cdot 3788 \pm 0.0870$ & $0.4778 \pm 0.0469$ & $0.4908 \pm 0.1170$ \\
\hline
\end{tabular}

variation was due to the additive component (a) $(P<0.001)$. There was also a dominance effect (b) but it was less significant $(P<0.05)$ than the additive effect. Partition of dominance effect demonstrated that the significant effect could be fully explained by directional dominance (b1) in the direction of decreasing IO, this is to say to increasing sensitivity.

\section{(b) Chromosome substitution assay}

The mean of 10 replicated tests for every genotype is presented in table 3 for both substitution assays. The parameter values and their significance are in table 4. It corresponds to the single degree of freedom contrasts of Mather and Jinks, described earlier. If we observe the effect of each individual chromosome, chromosome III had a very significant effect $(P<0.001)$ on the olfactory re-

Table 2 Partition of variance according to Hayman's model. (a) additive component, (b) dominant component, (b1) directional dominance, (b2) no directional dominance, (b3) dominant effect specific to individual crosses, (c) maternal effect and (d) other reciprocal effects

\begin{tabular}{lrcc}
\hline Source of variation & $\mathrm{df}$ & M.S. & $F$ \\
\cline { 2 - 4 }$-a$ & 6 & 0.3447 & $10 \cdot 71^{* * *}$ \\
$-b$ & 21 & 0.0490 & $1.97^{*}$ \\
$-b_{1}$ & 1 & 0.2552 & $15 \cdot 61^{*}$ \\
$b_{2}$ & 6 & 0.0455 & 1.42 \\
$-b_{3}$ & 14 & 0.0358 & 1.60 \\
$-c$ & 6 & 0.0285 & 1.27 \\
$-d$ & 15 & 0.0248 & 1.43 \\
- Block & 3 & 0.0337 & \\
Interactions & & & \\
$-a \times$ Block & 18 & 0.0322 & \\
$-b \times$ Block & 63 & 0.0249 & \\
$\bullet b_{1} \times$ Block & 3 & 0.0164 & \\
$-b_{2} \times$ Block & 18 & 0.0320 & \\
$-b_{3} \times$ Block & 42 & 0.0225 & \\
$-c \times$ Block & 18 & 0.0224 & \\
$-d \times$ Block & 45 & 0.0173 & \\
\hline
\end{tabular}

sponse differences and this effect was completely additive. None of the other major chromosomes showed any effect themselves. This fact was common in the two substitution sets.

Moreover, there are some significant interchromosomal interactions. First we will describe interaction among similar chromosomal combinations, this is to say $d \times d, d \times d \times d, h \times h$ and

Table 3 Mean values of the two sets of substitution assaies. Set $4-8$ between lines $45-1$ and $89-1$ and set $4-2$ between lines $45-1$ and 25-13. chromosomal combinations are named by three symbols, one for each chromosome, the first for chromosome I etc. ... When the symbol is a number it means homozygote for this chromosome, letter $X$ means heterozygote combination

\begin{tabular}{|c|c|c|c|}
\hline & $25-13 \times 45-1$ & & $89-1 \times 45-1$ \\
\hline genotype & & genotype & \\
\hline 222 & $0.4134 \pm 0.0487$ & 888 & $0.4021 \pm 0.0541$ \\
\hline $22 X$ & $0.3748 \pm 0.0885$ & $88 X$ & $0.2373 \pm 0.0572$ \\
\hline 224 & $0.1246 \pm 0.0265$ & 884 & $0.1721 \pm 0.0195$ \\
\hline $2 X_{2}$ & $0.3084 \pm 0.0754$ & $8 \times 8$ & $0.3584 \pm 0.0534$ \\
\hline $2 X X$ & $0.2325 \pm 0.0672$ & $8 X X$ & $0.3374 \pm 0.0520$ \\
\hline $2 \times 4$ & $0.2485 \pm 0.0615$ & $8 \times 4$ & $0.1086 \pm 0.0543$ \\
\hline 242 & $0.2605 \pm 0.0950$ & 848 & $0.3004 \pm 0.0750$ \\
\hline $24 X$ & $0.1335 \pm 0.0528$ & $84 X$ & $0.1764 \pm 0.0368$ \\
\hline 244 & $0.1147 \pm 0.0395$ & 844 & $0.2764 \pm 0.0890$ \\
\hline$X 22$ & $0.4195 \pm 0.0632$ & $X 88$ & $0.3899 \pm 0.0411$ \\
\hline$X 2 X$ & $0.2275 \pm 0.0494$ & $X 8 X$ & $0.4233 \pm 0.0539$ \\
\hline$X 24$ & $0.1903 \pm 0.0618$ & $X 84$ & $0.1882 \pm 0.0314$ \\
\hline$X X 2$ & $0.3080 \pm 0.0487$ & $X X 8$ & $0.4000 \pm 0.0521$ \\
\hline$X X X$ & $0.2593 \pm 0.0438$ & $\boldsymbol{X X X}$ & $0.1735 \pm 0.0543$ \\
\hline$X X 4$ & $0.1135 \pm 0.0439$ & $X \times 4$ & $0.1718 \pm 0.0500$ \\
\hline$X 42$ & $0.2095 \pm 0.0286$ & $X 48$ & $0.3183 \pm 0.0507$ \\
\hline$X 4 X$ & $0.1469 \pm 0.0408$ & $X 4 X$ & $0.0663 \pm 0.0204$ \\
\hline$X 44$ & $0.3231 \pm 0.0869$ & $X 44$ & $0.2837 \pm 0.0797$ \\
\hline 422 & $0.4135 \pm 0.0345$ & 488 & $0 \cdot 3248 \pm 0 \cdot 0440$ \\
\hline $42 X$ & $0.3250 \pm 0.0727$ & $48 X$ & $0.2762 \pm 0.0498$ \\
\hline 424 & $0.0645 \pm 0.0192$ & 484 & $0.2399 \pm 0.0711$ \\
\hline $4 \times 2$ & $0 \cdot 2790 \pm 0 \cdot 0528$ & $4 X 8$ & $0 \cdot 1879 \pm 0.0505$ \\
\hline $4 X X$ & $0.2187 \pm 0.0519$ & $4 X X$ & $0.1617 \pm 0.0372$ \\
\hline $4 \times 4$ & $0.2588 \pm 0.0479$ & $4 X 4$ & $0.1165 \pm 0.0289$ \\
\hline 442 & $0.2286 \pm 0.0547$ & 448 & $0.2890 \pm 0.0289$ \\
\hline $44 X$ & $0.1328 \pm 0.0533$ & $44 X$ & $0.2134 \pm 0 \cdot 0405$ \\
\hline 444 & $0.1678 \pm 0.0558$ & 444 & $0.1210 \pm 0.0151$ \\
\hline
\end{tabular}


Table 4 Values and signification of additive and dominant effect of the chromosomes as well as interchromosomal interactions

\begin{tabular}{|c|c|c|}
\hline & $25-13 \times 45-1$ & $89.1 \times 45-1$ \\
\hline$m$ & $0 \cdot 2235^{* * *}$ & $0.2532^{* * *}$ \\
\hline$d_{1}$ & 0.0048 & 0.0345 \\
\hline$d_{2}$ & 0.0305 & 0.0315 \\
\hline$d_{3}$ & $0 \cdot 1055^{* * *}$ & $0.0758 * * *$ \\
\hline$h_{1}$ & 0.0622 & 0.0418 \\
\hline$h_{2}$ & 0.0488 & -0.0603 \\
\hline$h_{3}$ & 0.0181 & -0.0274 \\
\hline$i_{12}$ & 0.0101 & -0.0322 \\
\hline$i_{13}$ & 0.0031 & -0.0124 \\
\hline$i_{23}$ & $0.0539 * *$ & 0.0029 \\
\hline$j_{1.2}$ & 0.0013 & 0.0061 \\
\hline$j_{1.3}$ & 0.0078 & -0.0535 \\
\hline$j_{2,3}$ & 0.0778 & -0.0006 \\
\hline$j_{2.1}$ & -0.0113 & -0.0375 \\
\hline$j_{3.1}$ & $-0.0766^{*}$ & -0.0168 \\
\hline$j_{3.2}$ & $-0.0841^{*}$ & 0.0044 \\
\hline $1_{12}$ & $-0.1237^{*}$ & 0.0512 \\
\hline $1_{13}$ & -0.1165 & -0.0184 \\
\hline $1_{23}$ & -0.0647 & 0.0841 \\
\hline$i_{123}$ & -0.0181 & $0.0486^{*}$ \\
\hline$j_{12.3}$ & 0.0021 & 0.0317 \\
\hline$j_{13.2}$ & 0.0054 & 0.0570 \\
\hline$j_{23.1}$ & 0.0318 & 0.0389 \\
\hline$j_{1.23}$ & -0.0071 & $0 \cdot 1007^{*}$ \\
\hline$j_{2.13}$ & -0.0568 & $0 \cdot 1896^{* * *}$ \\
\hline$j_{3.12}$ & $0.1524^{*}$ & 0.0506 \\
\hline $1_{123}$ & $0 \cdot 2116^{*}$ & $-0 \cdot 1508$ \\
\hline
\end{tabular}

*** $P<0.001 ;{ }^{* *} P<0.01 ;{ }^{*} P<0.05$.

$h \times h \times h$. In the set 2-4 there was a significant $(P<0.01)$ and positive $i 23$ interaction. This means an increasing of IO values when homozygous combinations of the chromosomes II and III of the same original line were put together. The $l 12$ interaction was significant $(P<0.05)$ and negative, this is to say $I O$ values decrease when heterozygous combinations of the chrumosomes I and II were together. The $l 123$ interaction was also significant $(P<0.05)$ but positive in the direction of increasing $\mathrm{IO}$ values when the three heterozygous were assembled. However this effect was not larger than the negative effect of the additive combination of double $l$ interactions and the total effect when the three heterozygous combinations for the I, II and III chromosomes were together was negative in the direction of increasing sensitivity.

In the set 8-4 only the interaction $i 123$ turned out significant $(P<0.05)$ and positive among $i$ and $l$ interactions.

Moreover, this kind of interactions, in the set 2-4 parameters $j 3.1, j 3.2$ and $j 3.12$ were significant $(P<0.05)$ and in the set $8-4, j 1.23(P<0.05)$ and j2.13 $(P<0.001)$ were significant.
The results of these two chromosomal analyses agree with those of diallel analysis, the additive effect of the chromosome III, and directional dominance with the positive $i$ interaction (endogamic depression) and the negative $l$ interaction (heterosis). The other kind of interactions, $j$, were not present in the diallel analysis because only homozygous combinations or heterozygous for the three chromosomes were obtained in the F1 generation.

\section{(c) Specificity analysis}

The results of the four synthetic lines A, B, C and $\mathrm{D}$ to six different chemicals are presented in fig. 2 .

First we wanted to study the kind of differences found to ethyl alcohol ( 8 per cent) among lines. The results obtained with different concentrations (fig. 2) allowed us to reject the idea of anosmia in line $\mathrm{D}$ because in all the lines response depended on concentration. An analysis of variance showed highly significant differences due to concentration $(P<0.001)$. Regression study (table 5$)$ showed a good adjustment of line $A$ to a regression line (deviation not significant) but it did not in line $D$ (deviation significant, $P<0.01$ ). Lines $B$ and $C$ showed a intermediate situation, as was expected of their origin (crossing one line with $\mathrm{IO}$ high an other with IO low). Differences were maximum in response to 10 per cent concentration, the nearest to tested concentration ( 8 per cent) in the first study.

Differences in response to ethyl alcohol between lines $A$ and $D$ can be summarised as follows: (a) line A was more sensitive than line D, because the same concentrations were more repellent for it than for line D and (b) the dose-response curve was linear in line $\mathrm{A}$ but it was not in line $\mathrm{D}$, which showed an increase of IO values at intermediate concentrations. Line B $(45-1 \times 89-1)$ and line $C(45-1 \times 25-133)$ were intermediates.

We are going to examine these differences found mainly between line $A$ and $D$ for the response to the other chemicals studied. Responses to methyl alcohol and ethyl acetate agreed with those of ethyl alcohol in sensitivity as well as in the curve shape. On the regresion analysis (table 5) line A showed a good adjustment to regression line in response to methyl alcohol but it did not in line $\mathrm{D}$. Line $\mathrm{A}$ in response to ethyl acetate showed the same behaviour, but line D gave a non significant regression line. This was due to the high value of deviation.

Response to acetone was very similar to that of ethyl alcohol. Differences in response to every 

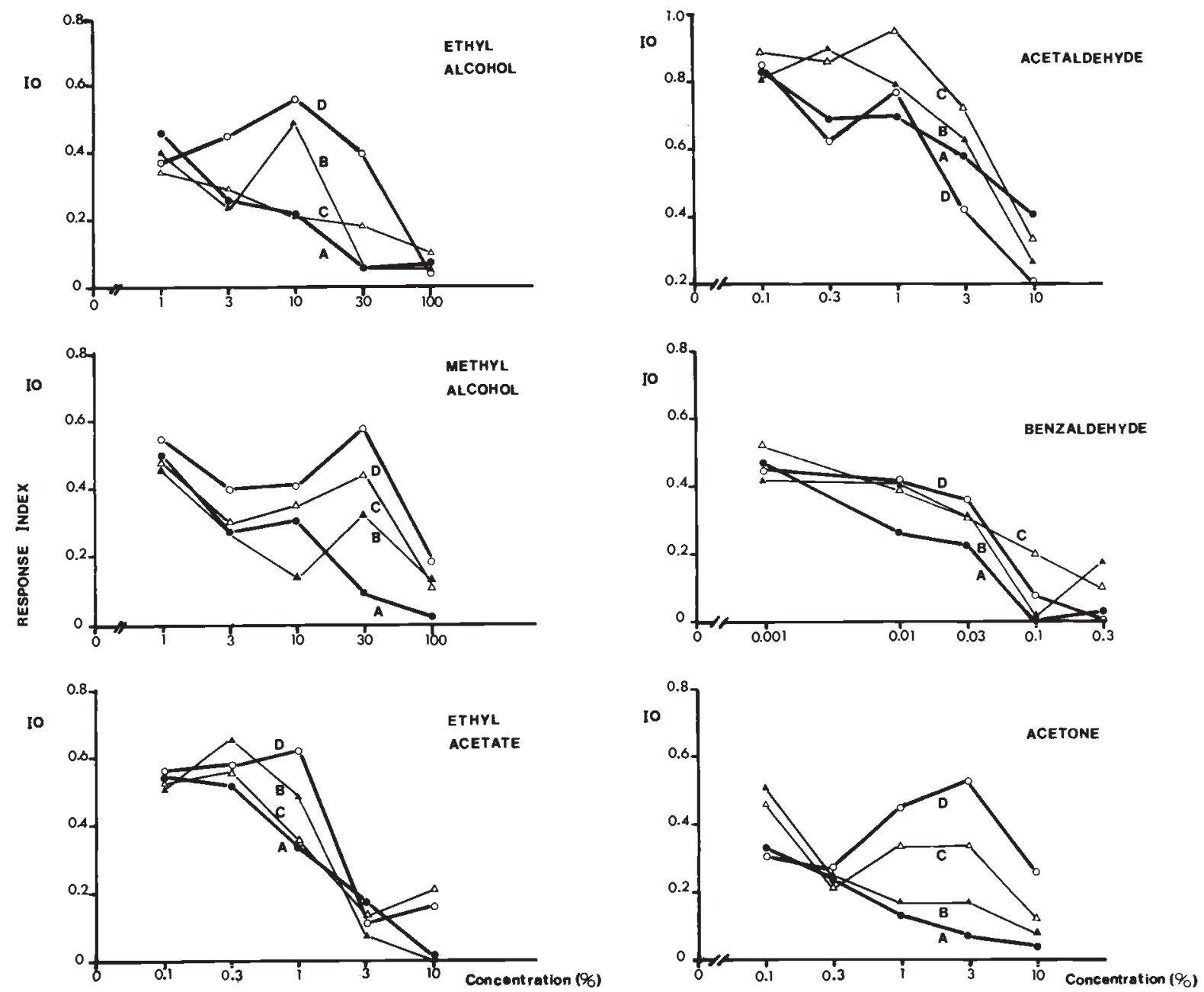

Figure 2 Dose-response curves to six odorants of four synthetic lines, $(0) \mathrm{A},() \mathrm{B},() \mathrm{C}$ and $(0) \mathrm{D}$. Concentrations are expressed on percentages in volume.

concentration were smaller than those of other chemicals and regression analysis did not give significant results, but the values went in the same direction as responses to ethyl alcohol, methyl alcohol and ethyl acetate.

Response to the two aldehydes tested agreed partially with the above results. The dose-response curve to acetaldehyde of line A showed a linear shape and line D a nonlinear shape, the same as the response to ethyl alcohol, but there were not the same differences in sensitivity. Response to benzaldehyde showed the same direction in response differences as that of ethyl alcohol but regression lines for $A$ and $D$ showed significant adjustment, however it was better for line $A$ than for $D$. In response to both aldehydes we can find the characteristics of response to ethyl alcohol in the response of the lines $\mathrm{B}$ and $\mathrm{C}$.

All of this pointed to the existence of differences in olfactory response of unspecific components, but the response could be added to other specific components in a different direction as in the case of aldehydes.

\section{DISCUSSION}

Genetic control of the olfactory response variation in a natural population has been revealed to be quite simple. The biometrical analysis using diallel crossing showed additive control and some directional dominance or overdominance in the direction to decrease IO values, as expected for a fitness 
Table 5 Analysis of adjustment to a regression line

\begin{tabular}{|c|c|c|c|c|c|c|c|c|}
\hline Line & $\begin{array}{l}\text { Source of } \\
\text { Variation }\end{array}$ & $\mathrm{df}$ & $\begin{array}{l}\text { Ethyl } \\
\text { Alcohol } \\
(\mathrm{MS})\end{array}$ & $\begin{array}{l}\text { Methyl } \\
\text { alcohol } \\
\text { (MS) }\end{array}$ & $\begin{array}{l}\text { Acetaldehyde } \\
\text { (MS) }\end{array}$ & $\begin{array}{l}\text { Benzaldehyde } \\
\text { (MS) }\end{array}$ & $\begin{array}{l}\text { Acetone } \\
\text { (MS) }\end{array}$ & $\begin{array}{l}\text { Ethyl } \\
\text { Acetate } \\
\text { (MS) }\end{array}$ \\
\hline \multirow[t]{4}{*}{ A } & Concentration & 4 & $0 \cdot 1084^{* *}$ & $0 \cdot 1478^{* *}$ & $0 \cdot 1024$ & $0 \cdot 1453^{* * * *}$ & $0.0591^{*}$ & $0 \cdot 2120^{* *}$ \\
\hline & Regression & 1 & $0 \cdot 3846^{* * *}$ & $0 \cdot 5291^{* * *}$ & $0.3706^{* *}$ & $0.5367^{* *}$ & $0 \cdot 2254$ & $0.8237^{* * *}$ \\
\hline & Deviation & 3 & 0.0164 & 0.0207 & 0.0130 & 0.0148 & 0.0037 & 0.0081 \\
\hline & Error & 15 & 0.0169 & 0.0299 & 0.0401 & 0.0088 & 0.0149 & 0.0356 \\
\hline \multirow[t]{4}{*}{ B } & Concentration & 4 & $0.1597^{* *}$ & $0 \cdot 0788^{* * *}$ & $0.2618^{* * *}$ & $0.1179^{*}$ & $0 \cdot 1061^{* *}$ & $0 \cdot 3343 * * *$ \\
\hline & Regression & 1 & 0.3064 & 0.1654 & 0.7943 & 0.2965 & $0.3433^{*}$ & 0.0128 \\
\hline & Deviation & 3 & $0 \cdot 1109^{*}$ & $0 \cdot 0500^{* *}$ & $0.0842^{*}$ & 0.0584 & 0.0271 & $0 \cdot 1081^{* *}$ \\
\hline & Error & 15 & 0.0263 & 0.0091 & 0.0212 & 0.0316 & 0.0131 & 0.0158 \\
\hline \multirow[t]{4}{*}{$\mathrm{C}$} & Concentration & 4 & 0.0352 & $0.0819^{* *}$ & $0 \cdot 2449^{* * *}$ & $0 \cdot 1090^{*}$ & 0.0667 & $0 \cdot 1441$ \\
\hline & Regression & 1 & $0.1397^{* *}$ & 0.1420 & 0.6241 & $0.4303^{* *}$ & 0.1149 & $0.4592^{* *}$ \\
\hline & Deviation & 3 & 0.0003 & $0 \cdot 0619^{*}$ & $0 \cdot 1185^{*}$ & 0.0019 & 0.0506 & 0.0390 \\
\hline & Error & 15 & 0.0152 & 0.0153 & 0.0300 & 0.0300 & 0.0378 & 0.0567 \\
\hline \multirow[t]{4}{*}{$\mathrm{D}$} & Concentration & 4 & $0.1531^{* *}$ & $0.1002^{*}$ & $0.2935^{* * *}$ & $0 \cdot 1696^{* *}$ & 0.0604 & $0 \cdot 2513^{* *}$ \\
\hline & Regression & 1 & $0 \cdot 2090$ & $0 \cdot 1273$ & $0.9354^{*}$ & $0.5525^{*}$ & 0.0110 & 0.6352 \\
\hline & Deviation & 3 & $0 \cdot 1344^{* *}$ & $0.0911^{*}$ & 0.0796 & 0.0419 & 0.0769 & 0.1231 \\
\hline & Error & 15 & 0.0192 & 0.0215 & 0.0266 & 0.0203 & 0.0278 & 0.0426 \\
\hline
\end{tabular}

*** $P<0.001$; ** $P<0.01 ; * P<0.05$.

trait (Breese and Mather, 1960; Kearsey and Kojima, 1967). This means in the direction of increasing sensitivity in the range of the responses that were studied.

The two chromosomal analyses agree with this first result and complement it. The major factor or factors controlling this olfactory response variation were located on chromosome III and their action was completely additive. This action was modulated by the genetic background as can be deduced from interchromosomal interactions. Some endogamic depression was found ( $i 23$ was significant in a substitution assay and $i 123$ in the other) in the direction of increasing IO values (to decrease sensitivity). Moreover, some significant heterosis has been found in one of the substitution sets ( $l 12$ and $l 123$ were significant) and the global direction was to decrease IO values, this is to say to increase sensitivity.

This genetic control indicates that the selection responses are the same as those obtained by Becker (1970) and Hoffman (1983) on natural populations, and it coincides with the additive effect of genes for variability to esters and ketones found by Fuyama (1978).

In this population some directional dominance for increased sensitivity seems to be protected as it can be expected from the use of olfactory sense to find food, mates and oviposition sites (i.e., Parsons, 1973). On the other hand, complete chromosomal analysis shows not only the chromosome or chromosomes responsible for major differences but interchromosomal relations.
The study of specific or unspecific nature of variation among extreme response lines pointed to a main unspecific factor depending on stimulus concentration. This fact agrees with correlation in response to different chemicals found in a first study of the population using isofemale lines. This unspecific component of olfactory response could correspond to different points in the chemosensorial pathway. It has been shown that unspecific systems are responsible for stimulus response after stimulus capture by specific receptor molecules even from transduction processes of chemical stimulus to electrical stimulus (Norris, 1981).

This work, besides Fuyama's isochromosomal work (1978), shows the existence of an important component of olfactory behaviour depending on autosomal chromosomes. Fuyama had already pointed out the existence of an unspecific factor of olfactory response on the chromosome III that modified the specific effect of chromosome II for esters and ketones, which he found in a natural population. This posibility has been confirmed by us. However, these two studies do not allow us to take general conclusions about the location of genes controlling specific and unspecific components of the complex olfactory behaviour.
Acknowledgements We wish to thank Dr A. Dominguez for valuable suggestions throughout this work and for her critical reading of the manuscript. 


\section{REFERENCES}

ALBORNOZ, J., DOMINGUEZ, A., ALCORTA, E. AND SANTIAGo, E. 1987. Chromosomal effects in egg laying of Drosophila melanogaster under different conditions. Heredity, 58, 457 462.

ACEVES-PIÑA, E. O. AND QUINN, W. G. 1979. Learning in normal and mutant Drosophila larvae. Science, 206, 93-95.

BECKER, H. J. 1970. The genetics of chemotaxis in Drosophila melanogaster. Selection for repellent insensitivity. Molec. Gen. Genet., 107, 194-200.

BREESE, E. L. AND MATHER, K. 1960. The organisation of polygenic activity within a chromosome in Drosophila. II. Viability. Heredity, 14, 375-399.

FUYAMA, Y, 1978. Behaviour Genetics of olfactory responses in Drosophila. II. an odorant-specific variant in a natural population of Drosophila melanogaster. Behav. Genet., 8, 399-414.

HAYMAN, B. I. 1954. The analysis of variance of diallel tables. Biom., 10, 235-244.

HOFFMAN, A. A. 1983. Bidirectional selection for olfactory response to acetaldehyde and ethanol in Drosophila melanogaster. Génét. Sél. Evol., 15, 501-518.
KEARSEY, M.J. AND KOJIMA, K. 1967. The genetic architecture of body weight and egg hatchability in Drosophila melanogaster. Genetics, 56, 23-37.

KIKUCHI, T. 1973. Specificity and molecular features of an insect attractant in a Drosophila mutant. Nature, 247, 3638.

MATHER, K. AND JINKS, J. L. 1971. Biometrical Genetics, Chapman and Hall, London.

NORRIS, D. M. 1981. Possible unifying principles in energy transduction in the chemical senses. In Norris, D. M. (ed.). Perception of Behavioural Chemicals, Biomedical Press, Elsevier/North Holland, Amsterdam.

PARSONS, P. A. 1973. Behavioural and Ecological Genetics: A study in Drosophila. Clarendon Press, Oxford.

RODRIGUES, V. AND SIDDIQI, O. 1978.. Genetic analysis of chemosensory pathway. Proc. Ind. Sci., 87B, 147-160.

SNEDECOR, G. W. AND COCHRAN, W. G. 1984. Métodos estadisticos, $10^{\circ}$ edición, C.E.C.S.A. ed., México.

SOKAL, R. R. AND ROHLF, F. J. 1979. In Blume, H. (ed.). Biometria. Principios y métodos estadisticos en la investigación biológica, Madrid.

VARGO, M. AND HIRSCH, J. 1986. Biometrical and chromosome analyses of lines of Drosophila melanogaster selected for central excitation. Heredity, 56, 19-24. 